\title{
On the characterization of host-guest inclusion complexes. Surface tension, calorimetry and molecular dynamics of cyclodextrins with a non-ionic surfactant
}

\author{
Ángel Piñeiro, ${ }^{*},{ }^{\dagger}$ Xavier Banquy, ${ }^{\dagger}$ Silvia Pérez-Casas, ${ }^{\dagger}$ Edgar Tovar, ${ }^{\dagger}$ \\ Abel García, ${ }^{\dagger}$ Alessandra Villa, ${ }^{\ddagger}$ Alfredo Amigo, ${ }^{\S}$ Alan E. Mark, ${ }^{\natural, \#}$ \\ and Miguel Costas ${ }^{*, t}$
}

Contribution from Laboratorio de Termofísica, Departamento de Fisicoquímica, Facultad de Química, Universidad Nacional Autónoma de México, Cd. Universitaria, México D.F. 04510, Mexico; Institute for Physical and Theoretical Chemistry, J.W. Goethe University, Max-von-Laue 7, 60439 Frankfurt, Germany; Departamento de Física Aplicada, Facultad de Física, Universidad de Santiago de Compostela, E-15782, Santiago de Compostela, Spain; Groningen Biomolecular Sciences and Biotechnology Institute (GBB), Department of Biophysical Chemistry, University of Groningen, Nijenborgh 4, 9747 AG Groningen, The Netherlands; School of Molecular and Microbial Sciences and the Institute for Molecular Biosciences, University of Queensland, St Lucia, 4072, Queensland, Australia.

\footnotetext{
${ }^{\dagger}$ Universidad Nacional Autónoma de México

‡ J.W. Goethe University

${ }^{\S}$ Universidad de Santiago de Compostela

१ University of Groningen

\# University of Queensland.
}

E-mail: fangel@servidor.unam.mx; costasmi@servidor.unam.mx

\section{Contents:}

1. Initial and final conformations after 10 ns of the seven MD trajectories for the inclusion complexes formed by $\alpha$-CD (Figure S1), $\beta$-CD (Figure S2) and $\gamma$-CD (Figure S3) with OGP. The number in the upper right corner of the initial structures corresponds to the labels used in Figure 4 of the article. The coordinates of all the structures are available upon request to the authors.

2. Movie showing a MD trajectory where the following sequence of spontaneous events is seen: $(i)$ an spontaneous release of an OGP molecule from the 1:2 complex with $\beta$ CD leading to a 1:1 species, (ii) the reappearance of the free OGP in the vicinity of the $\beta$-CD cavity, (iii) the competition between the two OGP molecules to occupy the cavity and (iv) the displacement of the original OGP molecule by the new one, producing another 1:1 complex. 
Figure S1
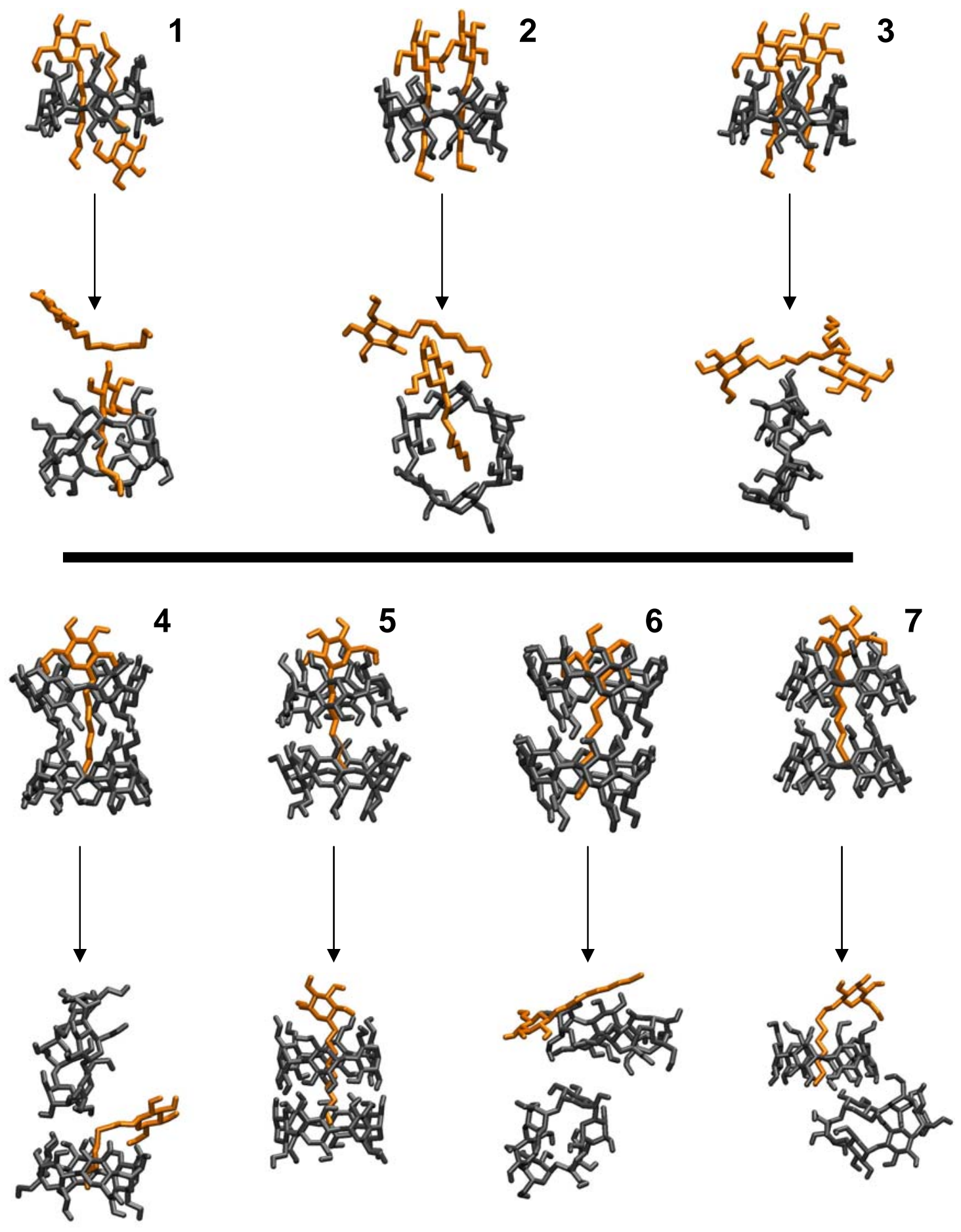
Figure S2
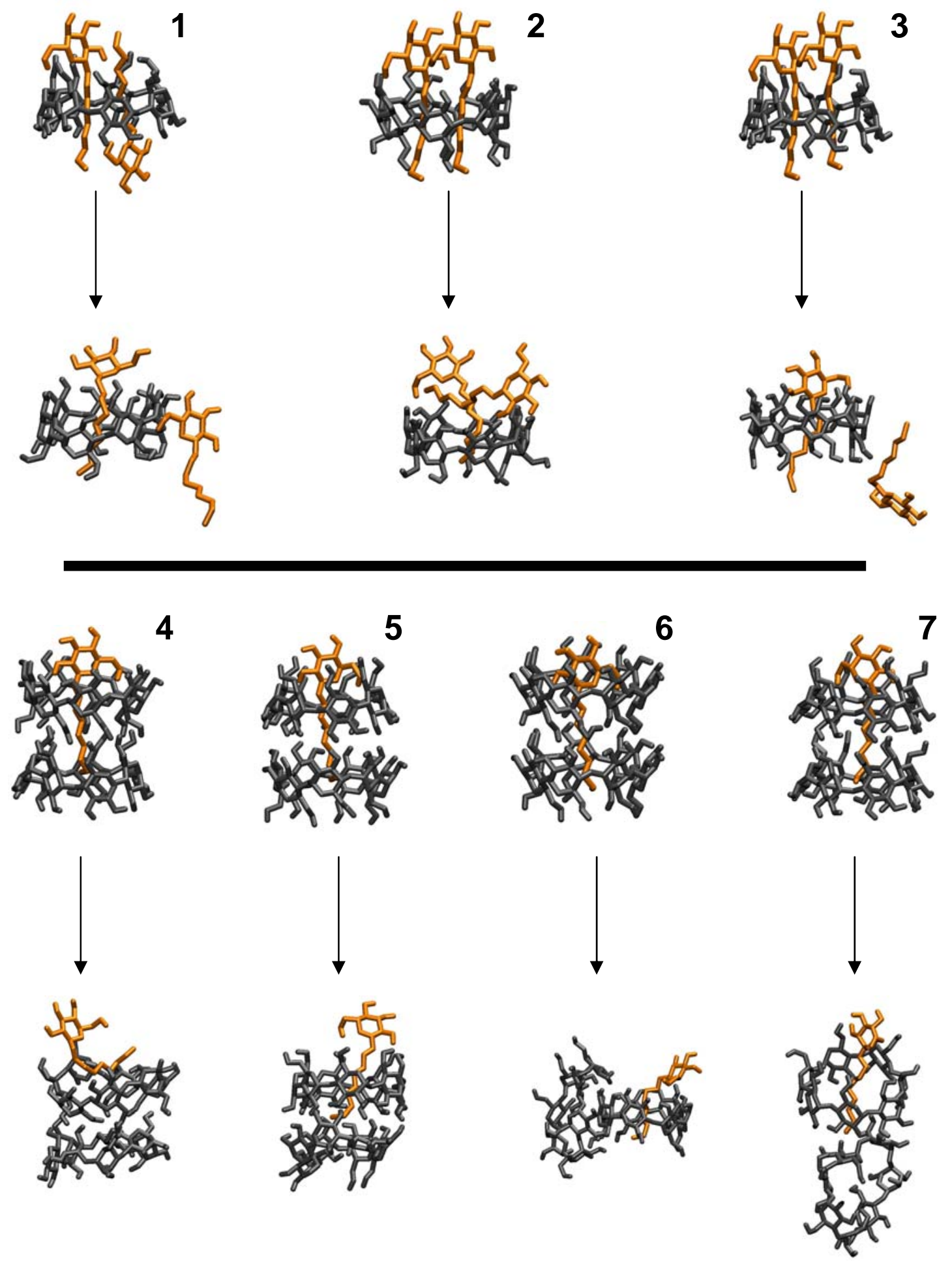
Figure S3
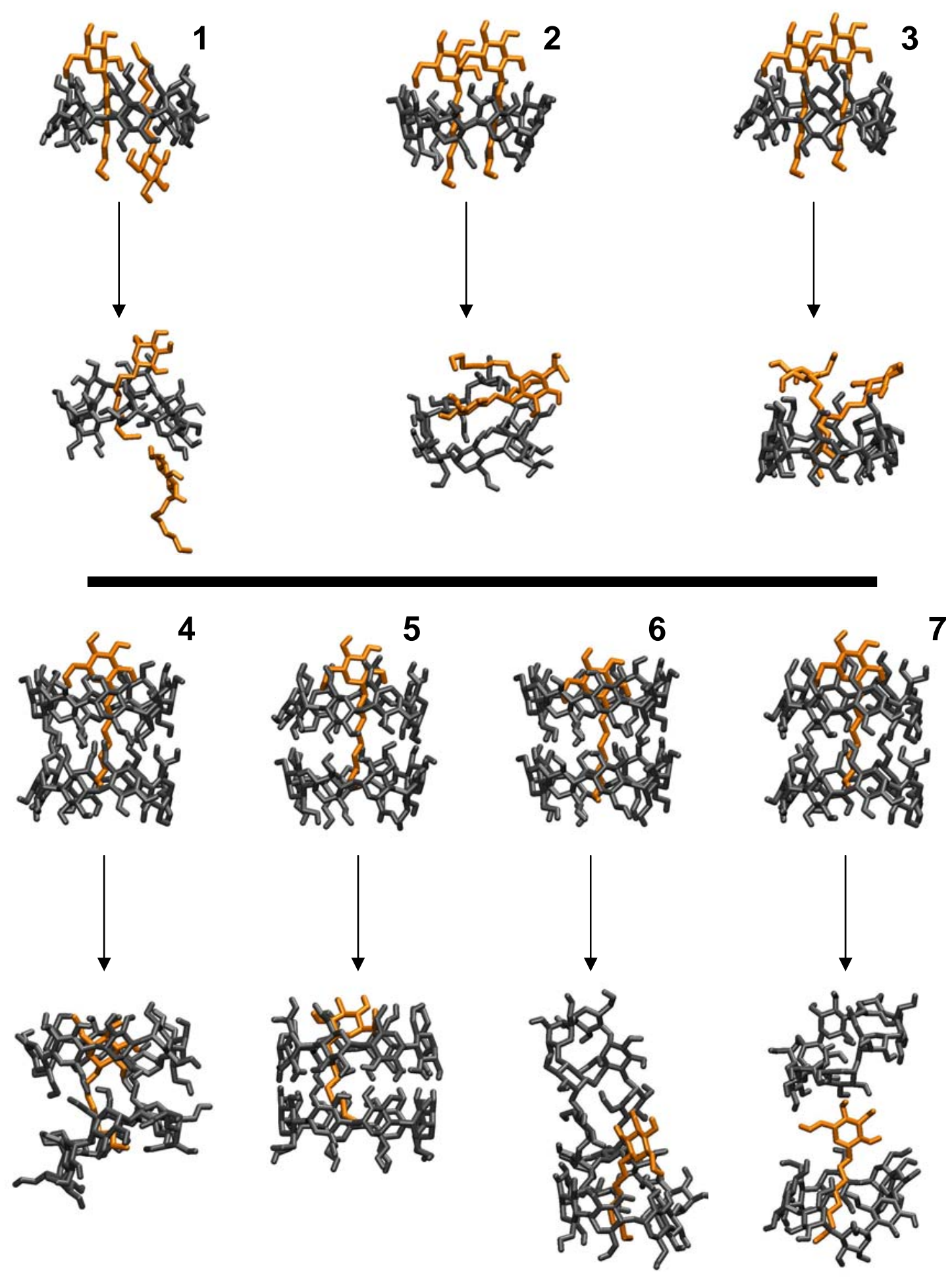